\title{
On the enumeration of uniquely reducible double designs
}

\author{
V. Fack ${ }^{1 \dagger}$, S. Topalova ${ }^{2 \ddagger}$ and J.Winne ${ }^{1}$ \\ ${ }^{1}$ Department of Applied Mathematics and Computer Science, Ghent University, Krijgslaan 281-S9, 9000 Ghent, Bel- \\ gium.E-mail:Veerle.Fack@UGent.be, Joost.Winne@UGent.be \\ ${ }_{2}^{2}$ Institute of Mathematics and Informatics, Bulgarian Academy of Sciences, P.O.Box 323, 5000 Veliko Tarnovo, Bul- \\ garia.E-mail: svetlana@moi.math.bas.bg
}

A double 2- $(v, k, 2 \lambda)$ design is a design which is reducible into two 2- $(v, k, \lambda)$ designs. It is called uniquely reducible if it has, up to equivalence, only one reduction. We present properties of uniquely reducible double designs which show that their total number can be determined if only the designs with non-trivial automorphisms are classified with respect to their automorphism group. As an application, after proving that a reducible 2- $(21,5,2)$ design is uniquely reducible, we establish that the number of all reducible 2-(21,5,2) designs is 1746461307.

Keywords: double design, projective plane, enumeration

\section{Preliminaries}

Let $V$ be a finite set of $v$ points and $\mathcal{B}$ a finite collection of $k$-element subsets of $V$, called blocks. $D=(V, \mathcal{B})$ is a $t-(v, k, \lambda)$ design if any $t$-subset of $V$ is contained in exactly $\lambda$ blocks of $\mathcal{B}$. Two designs are isomorphic if there exists a one-to-one correspondence between the point and block sets of the first design and the point and block sets of the second design, and if this one-to-one correspondence does not change the incidence. An automorphism is an isomorphism of the design to itself, i.e. a permutation of the points that transforms the blocks into blocks. The set of all automorphisms of a design form a group called its full group of automorphisms. Each subgroup of this group is a group of automorphisms of the design.

Each 2- $(v, k, \lambda)$ design determines the existence of 2- $(v, k, m \lambda)$ designs for any integer $m>1$. A 2$(v, k, m \lambda)$ design is called a quasimultiple of a $2-(v, k, \lambda)$ design. A quasimultiple $2-(v, k, m \lambda)$ is reducible into $m 2-(v, k, \lambda)$ designs if there is a partition of its blocks into $m$ subcollections each of which forms a $2-(v, k, \lambda)$ design. For $m=2$, quasimultiple designs are called quasidoubles and the reducible quasidouble designs are called doubles. We shall denote by $\left[D_{1} \mid D_{2}\right]$ a double design which can be reduced to the two designs $D_{1}$ and $D_{2}$. Here we will consider doubles of designs for which, up to isomorphism, only one design of its parameter set exists. So instead of $\left[D_{1} \mid D_{2}\right]$ we will often use the notation $[D \mid \varphi D]$, where

\footnotetext{
${ }^{\dagger}$ Corresponding author.

¥Partially supported by the Bulgarian National Science Fund under Contract I-1301/2003. 
the constituent design $\varphi D$ is obtained from $D$ by a permutation $\varphi$ of its points. By $G$ we denote the automorphism group of $D$; by $G_{\varphi}$ we denote the common subgroup of the automorphism groups of $D$ and $\varphi D$; by $\widehat{G}_{\varphi}$ we denote the automorphism group of the double design $[D \mid \varphi D]$.

In this paper we study uniquely reducible designs and show that the reducible $2-(21,5,2)$ designs are uniquely reducible. These are the doubles of the projective plane $P G(2,4)$ of order 4 , which is a unique $2-(21,5,1)$ design. Lower bounds on the number of doubles of projective planes can be found in [1] and [2]. We enumerate the reducible $2-(21,5,2)$ designs by constructing those which have non-trivial automorphisms. For other examples of enumerating designs which contain incidence structures see for instance [3], [4], [5].

\section{Uniquely reducible double designs}

Doubles can be reducible in more than one way. Two reductions $\left[D_{1} \mid D_{2}\right]$ and $\left[D_{3} \mid D_{4}\right]$ of one and the same double design are equivalent if and only if there exists some point permutation $\mu$ such that $D_{3}=\mu D_{1}$ and $D_{4}=\mu D_{2}$. A double which has, up to equivalence, only one reduction is uniquely reducible.

¿From now on we consider only uniquely reducible double designs. The following propositions give some properties of uniquely reducible designs, which will be useful for their enumeration.

Proposition 1 The $\operatorname{set}_{G}(\varphi)$ of all doubles of the form $[D \mid \psi D]$ which are isomorphic to $[D \mid \varphi D]$ is given by $\mathcal{C}_{G}(\varphi)=G \varphi G \cup G \varphi^{-1} G$. The number of such doubles is

$$
\left|\mathcal{C}_{G}(\varphi)\right|= \begin{cases}|G|^{2} /\left|G_{\varphi}\right| & \text { if } G \varphi G=G \varphi^{-1} G \\ 2|G|^{2} /\left|G_{\varphi}\right| & \text { otherwise, i.e. } G \varphi G \cap G \varphi^{-1} G=\emptyset .\end{cases}
$$

Proposition 2 If $G \varphi G=G \varphi^{-1} G$, then there exists $\omega \in \widehat{G}_{\varphi}$ such that $[D \mid \varphi D]=[D \mid \omega D]$. This $\omega$ transforms $D$ into $\varphi D$ and vice versa. If $\left|G_{\varphi}\right|=1$, then $\omega$ is of order 2 .

Corollary 3 If $\left|\widehat{G}_{\varphi}\right|=1$, then $\left|\mathcal{C}_{G}(\varphi)\right|=2|G|^{2}$.

Let $N_{i}\left(\operatorname{resp} . N_{i}^{\prime}\right)$ denote the number of classes $\mathcal{C}_{G}(\varphi)$ for which $\left|G_{\varphi}\right|=i$ and $G \varphi G \cap G \varphi^{-1} G=\emptyset$ (resp. $G \varphi G=G \varphi^{-1} G$ ), then

$$
v !=\sum_{\mathcal{C}_{G}(\varphi)}\left|\mathcal{C}_{G}(\varphi)\right|=2|G|^{2} N_{1}+|G|^{2} N_{1}^{\prime}+\sum_{i>1} \frac{2|G|^{2}}{i} N_{i}+\sum_{i>1} \frac{|G|^{2}}{i} N_{i}^{\prime}
$$

Let $N$ be the total number of non-isomorphic doubles of $D$, then

$$
N=N_{1}+N_{1}^{\prime}+\sum_{i>1} N_{i}+\sum_{i>1} N_{i}^{\prime}
$$

Constructing the doubles of $D$ with non-trivial automorphisms gives the numbers $N_{1}^{\prime}$ as well as $N_{i}$ and $N_{i}^{\prime}$ for all $i>1$. Equation (2) can be used to obtain the number $N_{1}$ of doubles of $D$ with trivial automorphisms. Equation (3) can be used to calculate the total number $N$ of doubles of $D$.

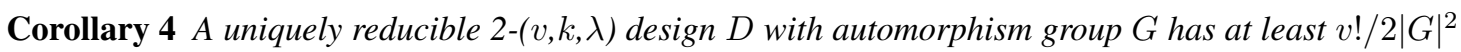
non-isomorphic doubles. 


\section{Enumeration of the reducible 2-(21,5,2) designs}

Proposition 5 A reducible 2-(21,5,2) design is uniquely reducible.

Proof: We give a sketch of the proof, which is based on the following observation.

Let $\left[D_{1} \mid D_{2}\right]$ be a double 2- $(v, k, 2 \lambda)$ design where $D_{1}$ and $D_{2}$ are isomorphic 2- $(v, k, \lambda)$ designs. Let this double design be reducible in two different ways. The blocks of $D_{1}$ and $D_{2}$ can then be partitioned into two collections $D_{1}^{a}$ and $D_{1}^{b}$, and $D_{2}^{a}$ and $D_{2}^{b}$, respectively, such that the blocks of $D_{1}^{a}$ and $D_{2}^{b}$ form a 2- $(v, k, \lambda)$ design, and the blocks of $D_{1}^{b}$ and $D_{2}^{a}$ also form a 2- $(v, k, \lambda)$ design.

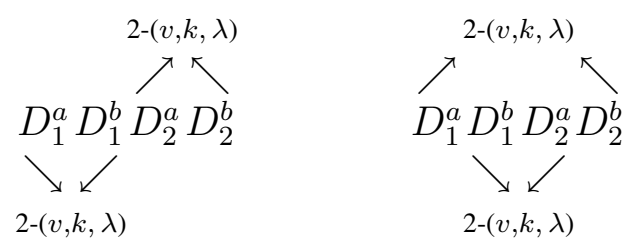

Without loss of generality we can demand the $a$ parts to be always larger than the $b$ parts, and $D_{1}^{b}$ and $D_{2}^{b}$ to have no blocks in common. Let $n$ be the number of blocks in $D_{1}^{b}$, and $a_{i}$ the number of blocks in $D_{1}^{b}$ containing point $i(i=1,2, \ldots, v)$. The following considerations can be made:

1. Any point is in the same number of blocks of $D_{1}^{a}$ and $D_{2}^{a}\left(D_{1}^{b}\right.$ and $\left.D_{2}^{b}\right)$.

2. Any pair of points is in the same number of blocks of $D_{1}^{a}$ and $D_{2}^{a}\left(D_{1}^{b}\right.$ and $\left.D_{2}^{b}\right)$.

3. If $D_{1}$ is a projective plane of order $q$ (i.e. a $2-\left(q^{2}+q+1, q+1,1\right)$ design), the following holds:

$$
\begin{aligned}
a_{i} & \neq 1 \quad ; i=1,2, \ldots, v \\
\sum_{i=1}^{v} a_{i} & =n(q+1) \\
\sum_{i=1}^{v} a_{i}^{2} & =n(n+q) \\
n & \leq \frac{q^{2}+q}{2}
\end{aligned}
$$

A careful study of all possible solutions of the system (4)-(7) for $q=4$, shows that a 2-(21,5,2) design cannot have two inequivalent reductions, i.e. it is uniquely reducible.

It follows from Corollary 4 that the number of the non-isomorphic doubles of the projective plane of order 4 is at least 1745944 200. To determine their exact number all designs with non-trivial automorphisms should be constructed.

In [6] all 2-(21,5,2) designs with automorphisms of odd prime orders were constructed, their number was determined to be 22998 and 4170 of them were found to be reducible. In [7] the authors constructed the designs with automorphisms of order 2 which transform each of the constituent 2-(21,5,1) designs into itself. 40485 such doubles were found, 305 of which have also an automorphism of odd prime order, 
so they were already counted among the 4170 doubles found above. This leaves only the reducible 2$(21,5,2)$ designs with automorphisms of order 2 which transform one of the constituent 2-(21,5,1) designs into the other and vice versa $\left(\left|G_{\varphi}\right|=1\right)$ to be constructed.

We generate all designs $[D \mid \varphi D]$, where $\varphi$ is a permutation of order 2. We first find all automorphisms of $D$ and then generate all possible permutations of order 2 in lexicographic order. As the automorphism group of the projective plane of order 4 is doubly transitive, we can fix one non-trivial orbit. Moreover a simple pruning condition allows to filter out many isomorphic copies, such that the final full isomorphism check does not filter much more. We checked the results by two different implementations. We construct 991957 non-isomorphic designs which have an automorphism of order 2 transforming the constituent designs into one another. We establish that for 984549 of them the order of the full group of automorphisms is 2 , and $\left|G_{\varphi}\right|=1$.

Having constructed all 1028899 doubles which possess non-trivial automorphisms, we use equations (2) and (3) to calculate the number of non-isomorphic designs without automorphisms, which turns out to be 1745432408 . The total number of 2-(21,5,2) doubles is 1746461307.

\section{References}

[1] D. Jungnickel. Quasimultiples of projective and affine planes. J. Geometry, 26:172-181, 1986.

[2] D. Jungnickel and K. Vedder. Simple quasidoubles of projective planes. Aequationes Mathematicae, 34:96-100, 1987.

[3] P. Kaski, P. Östergård, S. Topalova, and R. Zlatarski. Steiner triple systems of order 19 and 21 with subsystems of order 7,. Discrete Mathematics. To appear.

[4] C. Lam, S. Lam, and V.D. Tonchev. Bounds on the number of affine, symmetric and hadamard designs and matrices,. J. Combin. Theory A, 92:186-196, 2000.

[5] C. Lam, S. Lam, and V.D. Tonchev. Bounds on the number of hadamard designs of even order,. $J$. Combinatorial Designs, 9:363-378, 2001.

[6] S. Topalova. Enumeration of 2-(21,5,2) designs with automorphisms of an odd prime order. Diskretnii Analiz i Issledovanie Operatsii, 5(1):64-81, 1998. In Russian.

[7] S. Topalova and R. Zlatarski. Construction of doubles of the 2-(21,5,1) design with automorphisms of order 2. In Proceedings of the Ninth International Workshop on Algebraic and Combinatorial Coding Theory, Kranevo, Bulgaria, pages 379-383, 2004. 